\title{
Formations en « Instrumentation-Mesure-Qualité » pour des métiers à haute technologie
}

\author{
Annick Razet ${ }^{1}$ \\ Cnam, 61 Rue du Landy, 93210 La Plaine, Saint-Denis, France
}

\begin{abstract}
Résumé. Devant les exigences métrologiques spécifiques des métiers à haute technologie, et afin de répondre efficacement et de manière cohérence à l'émergence des besoins en termes de formation dans le domaine de l'instrumentation, de la mesure et de la qualité, le Conservatoire National des Arts et Métiers (Cnam) propose pour cette spécialité de dimension transversale forte, des modules d'enseignement (niveaux I à III, en formation initiale, en hors temps ouvrable, en formation continue ou en alternance) dans les grandes thématiques du Cnam (industrie, santé, environnement, qualité...).

Abstract. In view of the specific metrological requirements for trades in the field of high technologies and to respond effectively and consistently to the emerging needs in terms of training in the field of instrumentation, measurement and quality, the Conservatoire National des Arts et Métiers (Cnam) offers for this specialty of high transverse dimension, teaching modules (levels I to III, initial training, training outside working time, continuous training, apprenticeship training) in the major themes of the Cnam (industry, health, environment, quality ...).
\end{abstract}

\section{Introduction}

Devant l'émergence de nouveaux besoins en compétences dans le domaine de l'instrumentation, de la mesure et de la qualité pour les métiers à haute technologie, le Cnam propose pour cette spécialité, des formations allant $\mathrm{du}$ bac +2 au bac +8 avec différentes modalités : formation initiale, hors temps ouvrable, formation continue ou alternance.

\section{2 « Instrumentation-Mesure-Qualité » dans un contexte international}

Dans un contexte de concurrence internationale accrue, la qualité des mesures et la capacité d'attester celle-ci dans un cadre internationalement reconnu constituent un enjeu stratégique et sont un des axes forts d'accroissement de la compétitivité des entreprises. Réduire le coût de revient, améliorer les performances, réduire les risques et savoir garantir la qualité des produits sont une nécessité pour toute entreprise ou organisme. Les métiers de la mesure sont au cœur de cette action.

A l'aube de 2020, le monde est confronté à différents défis dans les domaines de la santé, l'industrie, la sécurité, l'environnement, l'énergie et le développement durable. Le caractère transverse de l'activité «Instrumentation-MesureQualité » conduit à la solliciter pour la plupart de ces défis.
Elle se doit d'accompagner l'innovation afin de soutenir les projets par des mesures traçables, caractérisées et fiables permettant une maîtrise des risques.

Les évolutions des secteurs à haute technologie ont conduit à un accroissement des exigences métrologiques impliquant la mesure dès la conception et tout au long du cycle de vie des produits.

\section{Evolution des compétences de la profession}

La mesure et l'instrumentation concernent l'ensemble des services techniques et scientifiques d'une entreprise, quel que soit son secteur d'activité (automobile, aéronautique, matériaux, pharmacie, chimie, cosmétique, agroalimentaire,...), et sont en interaction très forte avec les directions «qualité ». L'organisation en entreprise de ce domaine d'activités nécessite des ressources matérielles et humaines adaptées à la stratégie de l'entreprise.

La transversalité de l'activité « Instrumentation-MesureQualité »au sein des entreprises nécessite un personnel ayant des compétences dans un domaine spécifique (mesures physiques, génie électrique ou électronique, mécanique, etc.) auxquelles s'ajoutent des compétences associées à la maîtrise d'un processus de contrôle, mesure, essai, analyse (compétences pratiques, théoriques, organisationnelles). Ce large spectre de compétences se

1 annick.razet@cnam.fr 
décline entre les ingénieurs, les techniciens et les opérateurs de la manière suivante: organisationnelles (ingénieurs), théoriques (partagées par le technicien et l'ingénieur), et pratiques (partagées par l'opérateur et le technicien).

\section{Besoins en formation}

Compte tenu de la diversité des personnels en charge des activités métrologiques (opérateurs, techniciens et ingénieurs), l'offre de formation doit être adaptée aux profils des personnels concernés :

- «analyse de résultats de mesure et la méthode de mesure» pour l'ingénieur ou le technicien chargé du management du système de mesure et du contrôle produit

- «système de management» pour le cadre chargé du management de la fonction métrologie dans l'entreprise - «méthode de mesure ou d'étalonnage» pour le personnel opérationnel (opérateurs et techniciens).

\section{Offre de formation Cnam}

Créé en 1794, le Conservatoire national des arts et métiers (Cnam) est un grand établissement public dépendant du Ministère chargé de l'enseignement supérieur et dédié à la formation tout au long de la vie. Le Cnam propose un enseignement ouvert à tous, dans tous les domaines (parcours de formation ciblés sur les compétences recherchées par les entreprises, dans un très large éventail de filières-métiers), à différents niveaux (pour se perfectionner, améliorer ses compétences, acquérir une qualification ou un diplôme, se reconvertir), selon différentes formules (formations hors temps ouvrable, initiale, continue, en alternance), sur l'ensemble du territoire français et à l'étranger, étroitement lié au monde professionnel

Devant les exigences métrologiques spécifiques des métiers à haute technologie, et afin de répondre efficacement et de manière cohérence à l'émergence des besoins en termes de formation dans le domaine de l'instrumentation, de la mesure et de la qualité, le Cnam propose pour cette spécialité de dimension transversale forte, des modules d'enseignement allant du bac +2 au bac +8 , en formation initiale, en hors temps ouvrable, en formation continue ou en alternance dans les grandes thématiques du Cnam (industrie, santé, environnement, qualité...).

La spécificité de ces enseignements réside dans une approche de l'instrumentation et de la mesure au travers de la mise en œuvre, de la caractérisation métrologique et de l'exploitation d'une chaîne de mesure et d'analyse intégrant à la fois la maîtrise de la qualité et les enjeux en termes de stratégie et d'objectifs à atteindre grâce, notamment, à une part importante de travaux pratiques couvrant un large champ disciplinaire.

La figure 1 présente les formations Cnam spécifiques en Instrumentation-Mesure-Qualité ou ayant intégré des modules d'enseignement dans le domaine de l'instrumentation, de la mesure et de la qualité. L'évolution de ces formations accompagne l'évolution des technologies et du dispositif organisationnel des entreprises. Cette figure montre également le niveau de qualification (III à I) associé à chaque diplôme. Le niveau III correspond au bac +2 , le niveau II au bac $+3 / 4$, le niveau I au bac +5 .

Formations par unités d'enseignements (UE) capitalisables :

Diplôme d'Ingénieur Cnam «Mesure - Analyse» avec trois parcours: «Instrumentation Qualité», «Contrôle industriel», et «Analyse Chimique, Bioanalyse».

Le parcours «Instrumentation-qualité » met l'accent sur les outils théoriques et pratiques nécessaires à la conjugaison des aspects techniques dans le développement de l'instrumentation et de la mise en place de la démarche qualité au sein d'une entreprise ou dans un laboratoire, pour avoir et inspirer confiance dans les dispositifs et résultats d'équipements de contrôle, mesure et essais.

Le parcours «Contrôle industriel» est orienté vers le contrôle des procédés, l'exploitation d'installation mettant en œuvre l'informatique industrielle et les réseaux de terrain.

Le parcours « Analyse Chimique, Bioanalyse » est axé sur la mise en œuvre, efficiente et en adéquation avec les besoins des prescripteurs d'analyse, des diverses techniques d'analyse chimiques, physico-chimiques, biochimiques et biologiques mais aussi l'exploitation statistique et l'expression des résultats.

Titre RNCP niveau II " Responsable mesure, analyse, contrôle, qualité» avec deux parcours "Instrumentation Mesure » et « Analyse chimique et bioanalyse ».

Le parcours «Instrumentation mesure » est orienté vers la maîtrise des concepts et des outils de base de physique des capteurs, d'analyse et d'acquisition du signal, et de caractérisation métrologique d'une mesure et vers la déclinaison de ces outils dans un champ de spécialité donné afin d'obtenir et d'inspirer confiance dans les résultats obtenus.

Le parcours « Analyse chimique et bioanalyse» tend vers la maîtrise des techniques d'analyses chimiques, physicochimiques, biochimiques et biologiques ainsi que sur la méthodologie analytique (optimisation et validation des méthodes analytiques), et ce dans différents domaines.

Licence générale "Sciences de l'ingénieur en Matériaux, Instrumentation, Mécanique, Energétique (MIME)» parcours «Instrumentation Mesure Qualité ».

Pour acquérir les compétences sur les plans technique (outils et matériels de mesures), logistique, informatique (application de maintenance et de suivi des installations et équipements), scientifique (essais, analyses, contrôles et mesures qualité) et managérial (encadrement d'équipes techniques et de sous-traitants) pour mettre en œuvre et exploiter des processus de mesure, de contrôle ou d'essais en laboratoire ou en industrie.

Licence générale "Sciences pour l'ingénieur en Chimie Alimentation Santé (CAS)» parcours "Mesure, Analyse, Qualité ».

Pour acquérir les compétences dans la gestion et le contrôle qualité des analyses et des mesures (physiques, chimiques ou biologiques) en laboratoire ou sur une chaîne de fabrication dans des secteurs aussi variés que la chimie, la pharmacie, l'agro-alimentaire, l'environnement ou la santé. 
Figure 1. formations Cnam spécifiques en Instrumentation-Mesure-Qualité ou ayant intégré des modules d'enseignement dans le domaine de l'instrumentation, de la mesure et de la qualité

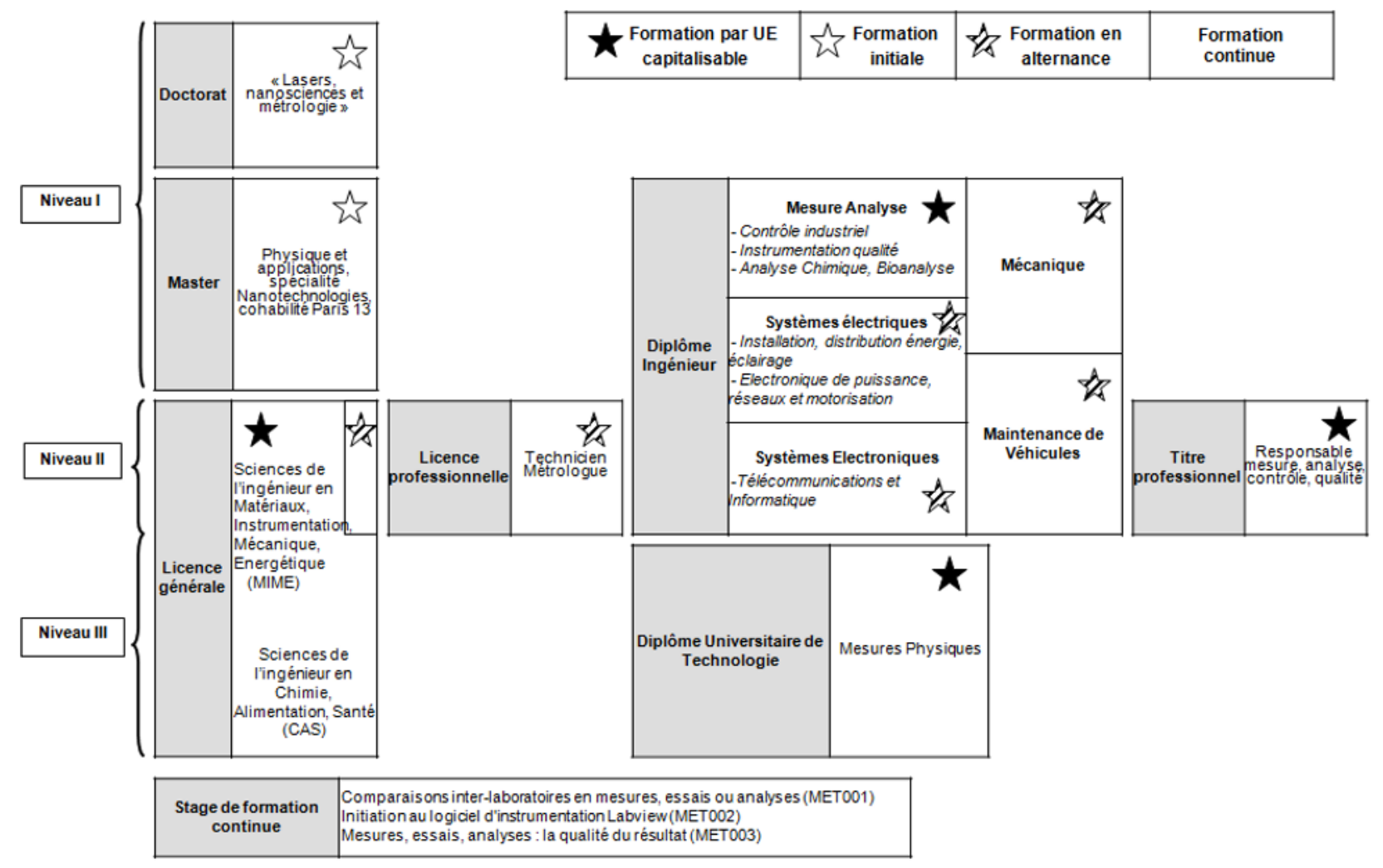

Diplôme universitaire de technologie « Mesures Physiques »

Pour acquérir les connaissances et les compétences nécessaires à un technicien supérieur en mesures et contrôles industriels pour tous les métiers liés à la mesure en recherche et développement ou en production.

\section{Formation initiale :}

Master «Physique et applications», spécialité «Nanotechnologies» en cohabilitation avec l'université Paris 13.

Les compétences visées concernent les aptitudes et l'initiation à la $\mathrm{R}$ et $\mathrm{D}$ dans les domaines couverts par le Master. Un approfondissement particulier est effectué, dans certains parcours, vers la maîtrise des moyens de mesure, de contrôle et d'essais.

\section{Doctorat « Lasers, nanosciences et métrologie »}

Former les futurs enseignants-chercheurs ou chercheurs en lasers, nanosciences et métrologie sur des sujets originaux et innovants.

\section{Formation en alternance :}

Licence professionnelle "Technicien Métrologue 》

Pour acquérir et consolider des compétences nouvelles en métrologie et se préparer à assumer des fonctions de responsabilité en matière de métrologie dans l'entreprise.
Licence générale «Sciences de l'ingénieur en Matériaux, Instrumentation, Mécanique, Energétique (MIME)» parcours "Instrumentation Mesure Qualité 》, troisième année (L3).

Diplôme d'Ingénieur Cnam en "Maintenance de Véhicules» Pour maîtriser les données technologiques les plus complexes entrant dans la maintenance des véhicules.

\section{Diplôme d'Ingénieur Cnam en "Mécanique »}

Formation conduisant aux connaissances et aux compétences liées à la prévision et à la conception de systèmes complexes en respectant une démarche qualité et en tenant compte de l'environnement.

Diplôme d'Ingénieur Cnam en "Systèmes électriques» parcours "Installation, distribution énergie, éclairage »

Pour être capable d'étudier et dimensionner des systèmes de distribution et de gestion de l'énergie électrique mais aussi d'éclairage, dans des domaines variés (bâtiment, industrie, voirie...), de concevoir des solutions innovantes qui intègrent notamment les contraintes environnementales.

Diplôme d'Ingénieur Cnam en "Systèmes électriques» parcours "Electronique de puissance, réseaux et motorisation »

Pour être capable de dimensionner et contrôler des systèmes de conversion d'énergie dans un souci d'efficacité énergétique, en intégrant les contraintes multi physique liées aux différents secteurs d'activité. 
Diplôme d'Ingénieur Cnam en "Systèmes Electroniques» parcours "Télécommunications et Informatique 》

Formation tenant compte des évolutions technologiques de l'électronique de demain avec une coloration dans le parcours en "Télécommunications", en "Embarqué et Durable" et en "Réseau et Sécurité".

\section{Formation continue :}

Actuellement au nombre de trois, ces stages sont orientés vers les comparaisons inter-laboratoires en mesures, essais ou analyses (MET001), l'Initiation au logiciel d'instrumentation Labview (MET002) et les Mesures, essais,analyses : la qualité du résultat (MET003). Le Cnam souhaite poursuivre le développement des stages interentreprises et des stages intra entreprises.

\section{Référence}

1. "Rapport final d'étude sur l'offre et les besoins de formation en métrologie", Ecole des mines de DOUAI pour le ministère de l'économie, des finances et de l'industrie (Mai 2010-février 2011). 